\title{
Geometric formulation of the uncertainty principle
}

\author{
G. M. Bosyk, ${ }^{1, *}$ T. M. Osán, ${ }^{2, \dagger}$ P. W. Lamberti, ${ }^{2, \dagger}$ and M. Portesi ${ }^{1, \S}$ \\ ${ }^{1}$ Instituto de Física La Plata (IFLP), CONICET, and Departamento de Física, Facultad de Ciencias Exactas, \\ Universidad Nacional de La Plata, 115 y 49, Casilla de Correo 67, 1900 La Plata, Argentina \\ ${ }^{2}$ Facultad de Matemática, Astronomía y Física, Universidad Nacional de Córdoba, and CONICET, \\ Avenida Medina Allende sin número, Ciudad Universitaria, X5000HUA Córdoba, Argentina
}

(Received 11 October 2013; published 10 March 2014)

\begin{abstract}
A geometric approach to formulate the uncertainty principle between quantum observables acting on an $\mathrm{N}$-dimensional Hilbert space is proposed. We consider the fidelity between a density operator associated with a quantum system and a projector associated with an observable, and interpret it as the probability of obtaining the outcome corresponding to that projector. We make use of fidelity-based metrics such as angle, Bures, and root infidelity to propose a measure of uncertainty. The triangle inequality allows us to derive a family of uncertainty relations. In the case of the angle metric, we recover the Landau-Pollak inequality for pure states and show, in a natural way, how to extend it to the case of mixed states in arbitrary dimension. In addition, we derive and compare alternative uncertainty relations when using other known fidelity-based metrics.
\end{abstract}

DOI: 10.1103/PhysRevA.89.034101

Introduction. The uncertainty principle is one of the major features of quantum mechanics, establishing a limitation on the predictability of incompatible observables. Uncertainty relations (URs) constitute the mathematical formulation of this principle. Variance-based URs, such as those of Heisenberg, Robertson, and Schrödinger [1], are the most popular ones; they exhibit a state-dependent lower bound for the product of the variances of a pair of noncommuting observables. Even though these kinds of URs allow us to form mental pictures useful for insights about quantum theory and also provide means for important quantitative predictions, they do not always capture the essence of the principle, as has been pointed out in Refs. [2,3]. Accordingly, a variety of alternative formulations have been proposed such as those using higher-order moments [4] or information-theoretic measures of ignorance which give entropic uncertainty inequalities [5]. The relevance of the study of URs relies to some extent on the fact that they are useful in several applications of quantum information as well as entanglement detection and quantum cryptography, among many others [6].

The geometric approach to quantum mechanics plays a fundamental role not only in foundational issues but also in applications of quantum information processing [7]. In order to contribute within this approach, we provide here a geometric formulation of the uncertainty principle. Our proposal is inspired by the Landau-Pollak inequality, introduced in timefrequency analysis [8] and later adapted to quantum mechanics by Maassen and Uffink [9].

This work is organized as follows: first, we review some known fidelity-based metrics as well as the Landau-Pollak inequality for pure states. Next, we introduce our major contributions: a proof of Landau-Pollak inequality for mixed quantum states and a geometric derivation of URs. Finally, some conclusions are drawn.

\footnotetext{
*gbosyk@ fisica.unlp.edu.ar

†tosan@famaf.unc.edu.ar

${ }^{\ddagger}$ lamberti@famaf.unc.edu.ar

§ portesi@fisica.unlp.edu.ar
}

PACS number(s): 03.65.Ta, 03.65.Aa, 02.50.-r, 03.67.-a

Theoretical background. Fidelity, defined by $F(\rho, \sigma)=$ $(\operatorname{Tr} \sqrt{\sqrt{\rho} \sigma \sqrt{\rho}})^{2}$, serves as a generalized measure of similarity between two quantum states represented by density matrices $\rho$ and $\sigma$ [10]. This quantity is not a metric but can be used to define metrics on the space of quantum states. We recall that a (true) metric is a function $d(\rho, \sigma)$ that verifies the following properties:

(i) non-negativity: $d(\rho, \sigma) \geqslant 0$, and $d(\rho, \sigma)=0$ iff $\rho=\sigma$,

(ii) symmetry: $d(\rho, \sigma)=d(\sigma, \rho)$,

(iii) triangle inequality: $d(\sigma, \rho)+d(\tau, \rho) \geqslant d(\sigma, \tau)$.

Now, we consider the following fidelity-based metrics that are used later to derive particular URs which, in turn, inspire alternative and more general relations.

(1) Angle metric:

It can be written in terms of fidelity as $[7,11]$

$$
d_{\mathrm{A}}(\rho, \sigma)=\arccos \sqrt{F(\rho, \sigma)} .
$$

In the case of two pure states $|\psi\rangle$ and $|\varphi\rangle$, the angle metric reduces to the Wootters metric [12]:

$$
d_{\mathrm{W}}(|\varphi\rangle,|\psi\rangle)=\arccos |\langle\varphi \mid \psi\rangle| .
$$

(2) Bures metric:

Written in terms of fidelity, it takes the form $[7,13]$

$$
d_{\mathrm{B}}(\rho, \sigma)=\sqrt{2-2 \sqrt{F(\rho, \sigma)}} .
$$

(3) Root-infidelity metric:

It can be written in terms of fidelity as [14]

$$
d_{\mathrm{RI}}(\rho, \sigma)=\sqrt{1-F(\rho, \sigma)} .
$$

Let us consider a quantum system with states belonging to an $N$-dimensional Hilbert space and two observables $\mathcal{A}$ and $\mathcal{B}$ with discrete nondegenerate spectra. Let $\left\{\left|a_{i}\right\rangle\right\}$ and $\left\{\left|b_{j}\right\rangle\right\}$ be the eigenbases of $\mathcal{A}$ and $\mathcal{B}$, respectively, and $|\Psi\rangle$ be a pure state of the quantum system. Then, the Landau-Pollak inequality (LPI) reads [3]

$$
\arccos \sqrt{P_{\mathcal{A} ; \Psi}}+\arccos \sqrt{P_{\mathcal{B} ; \Psi}} \geqslant \arccos c,
$$

where $\quad P_{\mathcal{A} ; \Psi}=\max _{i} p_{i}(\mathcal{A} ; \Psi)=\max _{i}\left|\left\langle a_{i} \mid \Psi\right\rangle\right|^{2} \in\left[\frac{1}{N}, 1\right]$ (and analogously for $\mathcal{B}$ ) and $c \equiv \max _{i, j}\left|\left\langle a_{i} \mid b_{j}\right\rangle\right| \in\left[\frac{1}{\sqrt{N}}, 1\right]$ 
is the so-called overlap between the eigenbases of the two observables. The LPI (5) is indeed an alternative formulation of the uncertainty principle for pure states [3]. Furthermore, it has been used to obtain entanglement criteria [15] and to improve the Maassen-Uffink entropic UR [16].

Now, we assert that LPI (5) is nothing but a consequence of the triangle inequality verified by the Wootters metric (2). Indeed, this inequality for the case of two arbitrary eigenstates $\left|a_{i}\right\rangle,\left|b_{j}\right\rangle$ of $\mathcal{A}$ and $\mathcal{B}$ respectively, and a given pure state $|\Psi\rangle$, reads

$$
\arccos \left|\left\langle a_{i} \mid \Psi\right\rangle\right|+\arccos \left|\left\langle b_{j} \mid \Psi\right\rangle\right| \geqslant \arccos \left|\left\langle a_{i} \mid b_{j}\right\rangle\right| .
$$

Choosing in particular those indices $i_{\max }$ and $j_{\max }$ that correspond to $\max _{i} p_{i}(\mathcal{A} ; \Psi)$ and $\max _{j} p_{j}(\mathcal{B} ; \Psi)$, we have

$$
\arccos \sqrt{P_{\mathcal{A} ; \Psi}}+\arccos \sqrt{P_{\mathcal{B} ; \Psi}} \geqslant \arccos \left|\left\langle a_{i_{\max }} \mid b_{j_{\max }}\right\rangle\right| .
$$

Thereby, taking into account that $\left|\left\langle a_{i_{\max }} \mid b_{j_{\max }}\right\rangle\right| \leqslant$ $\max _{i, j}\left|\left\langle a_{i} \mid b_{j}\right\rangle\right|=c$ and that arccosine is a decreasing function, it is straightforward to obtain the LPI (5).

With the purpose of obtainig URs valid for pure as well as for mixed quantum states, we extend the lines of reasoning employed to derive the inequality (5) to other metrics commonly used in quantum mechanics.

Geometric derivation of uncertainty relations. Now, we derive an extension of LPI to mixed quantum states acting on a Hilbert space of arbitrary dimension. Again the key of the proof relies upon the triangle inequality which for the angle metric and the triplet $\sigma, \rho, \tau$ gives

$$
\arccos \sqrt{F(\sigma, \rho)}+\arccos \sqrt{F(\tau, \rho)} \geqslant \arccos \sqrt{F(\sigma, \tau)} .
$$

Now, we use relation (8) for the operators $\sigma=\Pi_{i}^{\mathcal{A}}=\left|a_{i}\right\rangle\left\langle a_{i}\right|$ and $\tau=\Pi_{j}^{\mathcal{B}}=\left|b_{j}\right\rangle\left\langle b_{j}\right|$, which represent the rank-one projectors associated with the $i$ th outcome of $\mathcal{A}$ and the $j$ th outcome of $\mathcal{B}$, respectively, in such a way that for a system with density matrix $\rho$ one has

$$
F\left(\Pi_{i}^{\mathcal{A}}, \rho\right)=\operatorname{Tr}\left(\Pi_{i}^{\mathcal{A}} \rho\right)=p_{i}(\mathcal{A} ; \rho) .
$$

(and analogously for $\mathcal{B}$ ). Following the same lines of reasoning given previously to demonstrate LPI in the case of pure states, we find for mixed states

$$
\arccos \sqrt{P_{\mathcal{A} ; \rho}}+\arccos \sqrt{P_{\mathcal{B} ; \rho}} \geqslant \arccos c,
$$

where $P_{\mathcal{A} ; \rho}=\max _{i} p_{i}(\mathcal{A} ; \rho) \in\left[\frac{1}{N}, 1\right]$ (and analogously for $\mathcal{B})$ and the overlap in terms of the projectors reads $c=$ $\max _{i, j} \sqrt{\operatorname{Tr}\left(\Pi_{i}^{\mathcal{A}} \Pi_{j}^{\mathcal{B}}\right)}$.

Inequality (10) is an UR and it is the natural extension of LPI (5) to the case of mixed states in a Hilbert space of arbitrary dimension. To the best of our knowledge, this result was only formally proved for quantum states belonging to a Hilbert space of dimension 2 [17]. The extension of LPI to mixed states in arbitrary dimensions is one of the most important results of the present work.

We now show that a family of URs can be established in terms of fidelity-based metrics. The procedure to obtain these URs is inspired by the one followed to obtain inequality (10).

Let us start from fidelity-based metrics of the form

$$
d(\rho, \sigma)=f(F(\rho, \sigma))
$$

where $f(x)$ is a decreasing function for $x \in[0,1]$, with $f(x)=0$ iff $x=1$. Now, recalling the link between fidelity and probability given in Eq. (9), we propose as uncertainty measure for an observable $\mathcal{A}$ the following quantity:

$$
\mathcal{U}(\mathcal{A} ; \rho)=f\left(P_{\mathcal{A} ; \rho}\right) .
$$

Notice that this is a reasonable measure of uncertainty as it satisfies, by definition, the following basic properties:

(i) $\mathcal{U}(\mathcal{A} ; \rho) \geqslant 0$,

(ii) $\mathcal{U}(\mathcal{A} ; \rho)$ is decreasing in terms of $P_{\mathcal{A} ; \rho}$, that is, uncertainty decreases when one has more certainty about the predictability of $\mathcal{A}$,

(iii) The maximum of $\mathcal{U}(\mathcal{A} ; \rho)$ is attained at $P_{\mathcal{A} ; \rho}=\frac{1}{N}$, which leads to the uniform distribution; that is, uncertainty is maximum only when one has complete ignorance about the predictability of $\mathcal{A}$, and

(iv) $\mathcal{U}(\mathcal{A} ; \rho)=0$ iff $P_{\mathcal{A} ; \rho}=1$, that is, uncertainty vanishes only when one has certainty about the predictability of $\mathcal{A}$.

We now give our main result in the following proposition that establishes a geometric formulation of uncertainty principle:

Proposition. Let $\mathcal{A}$ and $\mathcal{B}$ be two observables with discrete nondegenerate spectra acting on an $N$-dimensional Hilbert space. Consider a quantum system described by a density operator $\rho$ and an uncertainty measure associated with the observables given in the form of Eq. (12). Then, the following UR holds:

$$
\mathcal{U}(\mathcal{A} ; \rho)+\mathcal{U}(\mathcal{B} ; \rho) \geqslant f\left(c^{2}\right),
$$

where $c^{2}=\max _{i, j} \operatorname{Tr}\left(\Pi_{i}^{\mathcal{A}} \Pi_{j}^{\mathcal{B}}\right)$, with $\Pi_{i}^{\mathcal{A}}$ and $\Pi_{j}^{\mathcal{B}}$ being the rank-one projectors associated with the $i$ th outcome of $\mathcal{A}$ and the $j$ th outcome of $\mathcal{B}$, respectively.

Proof. The triangle inequality fulfilled by Eq. (11) applied to the triplet $\Pi_{i}^{\mathcal{A}}, \Pi_{j}^{\mathcal{B}}, \rho$, leads to

$$
f\left(p_{i}(\mathcal{A} ; \rho)\right)+f\left(p_{j}(\mathcal{B} ; \rho)\right) \geqslant f\left(\operatorname{Tr}\left(\Pi_{i}^{\mathcal{A}} \Pi_{j}^{\mathcal{B}}\right)\right),
$$

where we made use of Eq. (9). Note that this inequality is valid for any pair of indices $i, j$. In particular, one can choose (separately) the indices $i_{\max }$ and $j_{\max }$ that correspond to the maximum probabilities $P_{\mathcal{A} ; \rho}$ and $P_{\mathcal{B} ; \rho}$, respectively. Using Eq. (12) we arrive at

$$
\mathcal{U}(\mathcal{A} ; \rho)+\mathcal{U}(\mathcal{B} ; \rho) \geqslant f\left(\operatorname{Tr}\left(\Pi_{i_{\max }}^{\mathcal{A}} \Pi_{j_{\max }}^{\mathcal{B}}\right)\right) .
$$

The proof concludes by taking into account that $\operatorname{Tr}\left(\Pi_{i_{\max }}^{\mathcal{A}} \Pi_{j_{\max }}^{\mathcal{B}}\right) \leqslant \max _{i, j} \operatorname{Tr}\left(\Pi_{i}^{\mathcal{A}} \Pi_{j}^{\mathcal{B}}\right)$ and that $f$ is decreasing.

We remark that, regardless of the explicit form of the uncertainty measure $\mathcal{U}$, our formulation captures the essence of the uncertainty principle, in the sense discussed in Refs. [2,3], due to the following reasons:

(i) the lower bound to the uncertainty sum is universal, that is, it is state-independent,

(ii) when $c<1$ the UR given by inequality (13) is nontrivial, that is, the uncertainty sum is strictly greater than zero, and

(iii) when $c=\frac{1}{\sqrt{N}}$ (complementary observables), certainty associated with one observable implies maximum ignorance about the other. 
Furthermore, it is worth noting that inequality (13) represents a family of URs.

Before presenting our conclusions, we particularize the UR given in the proposition for the case of the previously introduced fidelity-based metrics, and we compare the concomitant URs. In addition, we rephrase the results previously obtained for the angle metric and derive new results for Bures and root-infidelity metrics.

(1) Angle metric:

From Eqs. (1), (11), and (12), the corresponding uncertainty measure is $\mathcal{U}_{\mathrm{A}}(\mathcal{O} ; \rho)=\arccos \sqrt{P_{\mathcal{O} ; \rho}}$ for $\mathcal{O}=\mathcal{A}, \mathcal{B}$. Therefore, we reobtain inequality (10):

$$
\arccos \sqrt{P_{\mathcal{A} ; \rho}}+\arccos \sqrt{P_{\mathcal{B} ; \rho}} \geqslant \arccos c .
$$

Again we observe that this inequality is the natural extension of LPI (5) to the case of mixed states belonging to a Hilbert space of arbitrary dimension.

As we pointed out, this result has been proved for $N=2$ in Ref. [17], where the fact that $\arccos \sqrt{P_{\mathcal{O} ; \rho}}$ is concave in terms of $\rho$ was crucial for the demonstration; however, the same argument is not applicable when $N>2$ since that measure looses the concavity property.

(2) Bures metric:

From Eqs. (3), (11), and (12), the corresponding uncertainty measure is $\mathcal{U}_{\mathrm{B}}(\mathcal{O} ; \rho)=\sqrt{2-2 \sqrt{P_{\mathcal{O} ; \rho}}}$. Therefore, the following UR holds:

$$
\sqrt{1-\sqrt{P_{\mathcal{A} ; \rho}}}+\sqrt{1-\sqrt{P_{\mathcal{B} ; \rho}}} \geqslant \sqrt{1-c} .
$$

(3) Root-infidelity metric:

From Eqs. (4), (11), and (12), the corresponding uncertainty measure is $\mathcal{U}_{\mathrm{RI}}(\mathcal{O} ; \rho)=\sqrt{1-P_{\mathcal{O} ; \rho}}$. Therefore, the following UR holds:

$$
\sqrt{1-P_{\mathcal{A} ; \rho}}+\sqrt{1-P_{\mathcal{B} ; \rho}} \geqslant \sqrt{1-c^{2}} .
$$

Now, let us compare the three URs (14), (15), and (16) by focusing on the set of values of $P_{\mathcal{A} ; \rho}$ and $P_{\mathcal{B} ; \rho}$ allowed in each case. With this purpose, for each given $c \in\left[\frac{1}{\sqrt{N}}, 1\right]$, we define the sets

$$
\mathcal{D}_{\lambda, c}=\left\{\left(P_{\mathcal{A} ; \rho}, P_{\mathcal{B} ; \rho}\right) \in\left[\frac{1}{N}, 1\right]^{2}: P_{\mathcal{B} ; \rho} \leqslant g_{\lambda, c}\left(P_{\mathcal{A} ; \rho}\right)\right\},
$$

where $\lambda=\mathrm{A}, \mathrm{B}, \mathrm{RI}$ refers to angle, Bures, and root-infidelity metrics, respectively, and

$$
g_{\lambda, c}(P)= \begin{cases}1 & \text { if } \frac{1}{N} \leqslant P \leqslant c^{2}, \\ h_{\lambda, c}(P) & \text { if } c^{2} \leqslant P \leqslant 1,\end{cases}
$$

with $h_{\lambda, c}$ given in Table I (see Appendix). In Fig. 1 we show the sets $\mathcal{D}_{\lambda, c}$ for typical values of the overlap $c$.

TABLE I. Functions $h_{\lambda, c}$.

\begin{tabular}{lc}
\hline \hline Metric & $h_{\lambda, c}(P)$ \\
\hline$d_{\mathrm{A}}$ & $\left(\sqrt{1-P} \sqrt{1-c^{2}}+c \sqrt{P}\right)^{2}$ \\
$d_{\mathrm{B}}$ & $(\sqrt{P}+2 \sqrt{1-\sqrt{P}} \sqrt{1-c}+c-1)^{2}$ \\
$d_{\mathrm{RI}}$ & $P+2 \sqrt{1-P} \sqrt{1-c^{2}}+c^{2}-1$ \\
\hline \hline
\end{tabular}
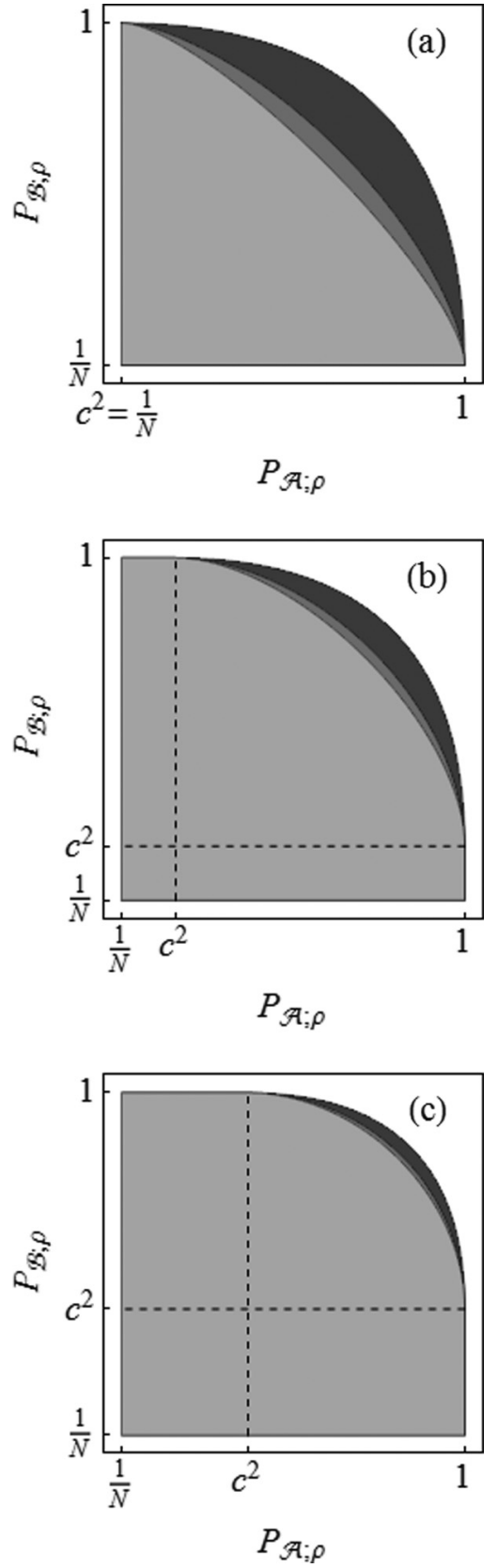

FIG. 1. Plots of the sets $\mathcal{D}_{\lambda, c}$ defined in Eq. (17) where $\lambda$ refers to angle metric (light gray), Bures metric (gray), and root-infidelity metric (dark gray), when $N=20$ and the overlap $c=\frac{1}{\sqrt{N}}(\mathrm{a}), \sqrt{0.2}$ (b), and $\sqrt{0.4}$ (c).

It can be seen that the following ordering among the sets holds for every $c$ :

$$
\mathcal{D}_{\mathrm{A}, c} \subseteq \mathcal{D}_{\mathrm{B}, c} \subseteq \mathcal{D}_{\mathrm{RI}, c} .
$$

This implies that inequality (14), derived from the angle metric, is the tightest one. However, two limiting cases arise where the three sets are equal. One case is that in which one has certainty about one observable: if $P_{\mathcal{A} ; \rho}=1$, then $P_{\mathcal{B} ; \rho} \leqslant g_{\lambda, c}(1)=c^{2}$ for any metric; and analogously interchanging $\mathcal{A}$ and $\mathcal{B}$. The other case is trivial: when $c=1$, the three sets are the whole square $\left[\frac{1}{N}, 1\right]^{2}$; that is, there is no restriction coming from URs.

Concluding remarks. We present a geometric approach to formulate the uncertainty principle. We obtain a family of 
TABLE II. Variable $\xi$ and coefficients of the quadratic polynomial in (A1), for angle, Bures, and root-infidelity metrics.

\begin{tabular}{lccc}
\hline \hline Metric & $\xi$ & $\alpha_{1}$ & $\alpha_{0}$ \\
\hline$d_{\mathrm{A}}$ & $\sqrt{1-P_{\mathcal{B} ; \rho}}$ & $2 c \sqrt{1-P_{\mathcal{A} ; \rho}}$ & $c^{2}-P_{\mathcal{A} ; \rho}$ \\
$d_{\mathrm{B}}$ & $\sqrt{2-2 \sqrt{P_{\mathcal{B} ; \rho}}}$ & $2 \sqrt{2-2 \sqrt{P_{\mathcal{A} ; \rho}}}$ & $2\left(c-\sqrt{P_{\mathcal{A} ; \rho}}\right)$ \\
$d_{\mathrm{RI}}$ & $\sqrt{1-P_{\mathcal{B} ; \rho}}$ & $2 \sqrt{1-P_{\mathcal{A} ; \rho}}$ & $c^{2}-P_{\mathcal{A} ; \rho}$ \\
\hline \hline
\end{tabular}

uncertainty relations, inequality (13), that depend on fidelitybased metrics. In particular, when we make use of the angle metric between quantum states, a natural generalization of the Landau-Pollak inequality to mixed states in arbitrary dimensions is obtained in relation (14). Furthermore, we find two additional uncertainty relations, inequalities (15) and (16), derived from Bures and root-infidelity metrics, respectively. These relations are seen to be weaker than the Landau-Pollak inequality. As a consequence of these findings, the question is raised of whether the angle metric leads to the tightest uncertainty relation when compared to an uncertainty relation derived from any arbitrary fidelity-based metric, within our approach. This observation deserves further study. Moreover, it remains open the problem of how to extend our main result (13), demonstrated for discrete nondegenerate observables, to the most general case of positive-operator valued measures.

Acknowledgments. M.P. and G.M.B. are grateful to SPU for financial support through the mobility program Inter-U and acknowledge warm hospitality at FaMAF-UNC. This work has been partially supported by PIP 1177/09 from CONICET. P.W.L. and T.M.O. are grateful to SECyT-UNC for financial support.

\section{APPENDIX: SETS OF ALLOWED VALUES OF $P_{\mathcal{A} ; \rho}$ AND $P_{\mathcal{B} ; \rho}$}

Here we show how to obtain the sets (17) of allowed values of $P_{\mathcal{A} ; \rho}$ and $P_{\mathcal{B} ; \rho}$ for angle, Bures, and root-infidelity metrics. The URs (14), (15), and (16) can be written as

$$
\xi^{2}+\alpha_{1} \xi+\alpha_{0} \geqslant 0
$$

where the variable $\xi$, and the coefficients $\alpha_{1}$ and $\alpha_{0}$ are given in Table II for each metric.

In all three cases the quadratic polynomial has two roots, $\xi_{ \pm}$, being $\xi_{-}$always negative while $\xi_{+}$can take either sign. If $P_{\mathcal{A} ; \rho} \leqslant c^{2}$ then $\xi_{+} \leqslant 0$ and $P_{\mathcal{B} ; \rho} \leqslant 1$; otherwise, $\xi_{+} \geqslant 0$ and $P_{\mathcal{B} ; \rho} \leqslant h_{\lambda, c}\left(P_{\mathcal{A} ; \rho}\right)$ with $h_{\lambda, c}$ given in Table I.
[1] W. Heisenberg, Z. Phys. 43, 172 (1927); H. P. Robertson, Phys. Rev. 34, 163 (1929); E. Schrödinger, Sitzungsber. Preuss. Akad. Wiss., Phys. Math. K1. 19, 296 (1930).

[2] D. Deutsch, Phys. Rev. Lett. 50, 631 (1983).

[3] J. B. M. Uffink, Ph.D. thesis, University of Utrecht, 1990 (unpublished).

[4] J. C. Angulo, J. Phys. A 26, 6493 (1993); Phys. Rev. A 50, 311 (1994); J. S. Dehesa, S. López-Rosa, and D. Manzano, in Statistical Complexities: Application to Electronic Structure, edited by K. D. Sen (Springer, Berlin, 2010); S. Zozor, M. Portesi, P. Sánchez-Moreno, and J. S. Dehesa, Phys. Rev. A 83, 052107 (2011).

[5] I. Bialynicki-Birula and J. Mycielski, Commun. Math. Phys. 44, 129 (1975); M. Krishna and K. R. Parthasarathy, Sankhya: Indian J. Stat., Ser. A 64, 842 (2002); G. C. Ghirardi, L. Marinatto, and R. Romano, Phys. Lett. A 317, 32 (2003); I. Bialynicki-Birula, Phys. Rev. A 74, 052101 (2006); S. Zozor and C. Vignat, Physica A (Amsterdam, Neth.) 375, 499 (2007); S. Zozor, M. Portesi, and C. Vignat, ibid. 387, 4800 (2008); A. E. Rastegin, J. Phys. A: Math. Theor. 43, 155302 (2010); I. Bialynicki-Birula and L. Rudnicki, in Statistical Complexity, edited by K. D. Sen (Springer, Berlin, 2011), Chap. 1; Y. Huang, Phys. Rev. A 83, 052124 (2011); M. Jafarpour and A. Sabour, ibid. 84, 032313 (2011); A. Luis, ibid. 84, 034101 (2011); A. E. Rastegin, Int. J. Theor. Phys. 51, 1300 (2012); G. M. Bosyk, M. Portesi, and A. Plastino, Phys. Rev. A 85, 012108 (2012); Z. Puchała, L. Rudnicki, and K. Życzkowski, J. Phys. A: Math. Theor. 46, 272002 (2013); S. Zozor, G. M. Bosyk, and M. Portesi, ibid. 46, 465301 (2013).

[6] H. F. Hofmann and S. Takeuchi, Phys. Rev. A 68, 032103 (2003); O. Gühne, Phys. Rev. Lett. 92, 117903 (2004); V. Giovannetti, Phys. Rev. A 70, 012102 (2004); O. Gühne and M. Lewenstein, ibid. 70, 022316 (2004); J. M. Renes and J. C. Boileau, Phys. Rev. Lett. 103, 020402 (2009); M. Berta,
M. Christandl, R. Colbeck, J. M. Renes, and R. Renner, Nat. Phys. 6, 659 (2010); S. Wehner and A. Winter, New J. Phys. 12, 025009 (2010); M. Tomamichel and R. Renner, Phys. Rev. Lett. 106, 110506 (2011); P. J. Coles, R. Colbeck, L. Yu, and M. Zwolak, ibid. 108, 210405 (2012); Y. Huang, Phys. Rev. A 86, 024101 (2012); Nelly Huei Ying Ng, M. Berta, and S. Wehner, ibid. 86, 042315 (2012); G. M. Bosyk, M. Portesi, F. Holik, and A. Plastino, Phys. Scr. 87, 065002 (2013); M. Tomamichel and E. Hänggi, J. Phys. A: Math. Theor. 46, 055301 (2013).

[7] I. Bengtsson and K. Życzkowski, Geometry of Quantum States: An Introduction to Quantum Entanglement (Cambridge University Press, Cambridge, 2006).

[8] H. J. Landau and H. O. Pollak, Bell Syst. Tech. J. 40, 65 (1961).

[9] H. Maassen and J. B. M. Uffink, Phys. Rev. Lett. 60, 1103 (1988).

[10] A. Uhlmann, Rep. Math. Phys. 9, 273 (1976); R. Jozsa, J. Mod. Opt. 41, 2315 (1994).

[11] M. A. Nielsen and I. L. Chuang, Quantum Computation and Quantum Information (Cambridge University Press, Cambridge, 2010).

[12] W. K. Wootters, Phys. Rev. D 23, 357 (1981); A. Majtey, P. W. Lamberti, M. T. Martín, and A. Plastino, Eur. Phys. J. D 32, 413 (2005).

[13] D. Bures, Trans. Amer. Math. Soc. 135, 199 (1969).

[14] A. Gilchrist, N. K. Langford, and M. A. Nielsen, Phys. Rev. A 71, 062310 (2005).

[15] J. I. de Vicente and J. Sánchez-Ruiz, Phys. Rev. A 71, 052325 (2005); T. Miyadera and H. Imai, ibid. 76, 062108 (2007); Y. Huang, ibid. 82, 012335 (2010).

[16] J. I. de Vicente and J. Sánchez-Ruiz, Phys. Rev. A 77, 042110 (2008); G. M. Bosyk, M. Portesi, A. L. Plastino, and S. Zozor, ibid. 84, 056101 (2011).

[17] J. L. de Vicente, Ph.D. thesis, Universidad Carlos III de Madrid, 2008 (unpublished). 